\title{
Dynamical Models of the Maser in NGC 4258
}

\author{
J. M. Moran \\ Harvard-Smithsonian Center for Astrophysics, 60 Garden St, \\ Cambridge, MA 02138, USA
}

\section{Introduction}

The presence of two quasars symmetrically displaced from the nucleus of the galaxy NGC 4258 (Pietsch et al. 1994) has given rise to some speculation that they might have some dynamical connection with the high-velocity maser emission seen in the nucleus (Burbidge 1996; Ozernoy 1996). The case that the masers define a simple Keplerian disk is very compelling; nonetheless, it is instructive to investigate whether any other dynamical models could fit the available data.

The evidence that the masers define a thin Keplerian disk can be found in Miyoshi et al. (1995) and Moran et al. (1995). Some of the relevant data are shown elsewhere in this volume (Fig. 2 of Greenhill's paper). The basic analysis is as follows. The features near the systemic velocity of $470 \mathrm{~km} \mathrm{~s}^{-1}$ show a linear dependence of line-of-sight velocity versus distance along the major axis, while the redshifted and blueshifted high-velocity features show a nearly Keplerian dependence with distance. For a Keplerian disk viewed edge on, the line-of-sight velocity with respect to the systemic velocity (for the case where the central mass is much greater than the disk mass) is given by

$$
v_{z}=\sqrt{\frac{G M}{R}} \sin \phi,
$$

where $M$ is the central mass, $G$ is the gravitational constant, $R$ is the radius from the center of mass, and $\phi$ is angle formed by the lines from the central mass to the observer and from the central mass to any maser spot. For small angles, $\phi=x / R$, where $x$ is the transverse offset from the central mass, so that

$$
v_{z}(\text { systemic })=\sqrt{\frac{G M}{R^{3}}} x .
$$

Hence, the slope of $v_{z}$ versus $x$ defines the enclosed mass density, $4 \times 10^{9} M_{\odot} \mathrm{pc}^{-1}$ in the case of NGC 4258, but does not allow for $M$ and $R$ to be determined independently. The Keplerian wings imply that $\sin \phi$ is constant for the high-velocity features. Since the high-velocity features show no $\left(<0.2 \mathrm{~km} \mathrm{~s}^{-1}\right)$ centripetal acceleration (Greenhill et al. 1995; Herrnstein et al. 1996), $\phi$ must be close to $90^{\circ}$. Hence,

$$
v_{z}(\text { high velocity })=\sqrt{\frac{G M}{x}} .
$$




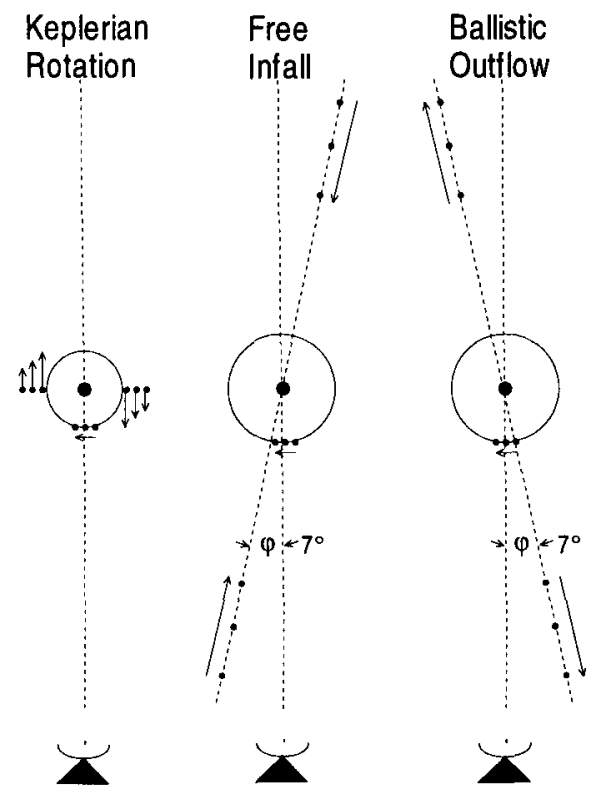

Figure 1. Three models that are consistent with the measurements of the positions, velocities, velocity drifts, and proper motions of the masers in NGC 4258. Left: The standard model of an edge-on disk in Keplerian motion. The circle denotes the inner boundary of maser emission with radius $R=0.13 \mathrm{pc}$. Center: Free infall of high-velocity masers. Right: Ballistic outflow of high-velocity masers with a terminal velocity of zero. In the infall and outflow models, the systemic masers still define a circumnuclear disk, but with slightly larger size and central mass than for the simple Keplerian model.

The fit to the Keplerian portion of the velocity curve is very good, with a fractional velocity deviation $\Delta v_{z} / v_{z}<0.003$. The disk is also very thin, with a thickness-to-radius ratio of $<0.0025$, although it is slightly warped (Herrnstein, Greenhill, \& Moran 1996). The well-defined linear relation between systemic velocity and position means that the fractional annular thickness of the systemic group of masers is only about 0.1 . The systemic features drift at a rate of about $9 \mathrm{~km} \mathrm{~s}^{-1} \mathrm{yr}^{-1}$, which is consistent with the model for a distance of about $6.4 \mathrm{Mpc}$. For this distance the central mass is $3.6 \times 10^{7} M_{\odot}$ and the radius of the systemic features is $0.13 \mathrm{pc}$. Preliminary analysis of proper-motion data (Herrnstein 1996) show that the blueshifted high-velocity features have proper motions of less than $15 \mu \mathrm{arcsec} \mathrm{yr}^{-1}$, but the systemic features move at about $30 \mu \operatorname{arcsec} \mathrm{yr}^{-1}$, both with respect to the redshifted high-velocity features. These data all argue persuasively for a simple model of Keplerian motion. The model is sketched in Fig. 1 (left). 


\section{The High-Velocity Features as Ballistic Outflow}

The question arises as to whether the high-velocity features could be tracing outflowing material. For ballistic motion. the outflow velocity would be

$$
v=\sqrt{\frac{2 G M^{\star}}{R}}+v_{\infty}
$$

where $v_{\infty}$ is the terminal velocity, which, from the data for NGC 4258 , is zero. Hence, the line of-sight velocity versus projected distance would be

$$
v_{z}=\sqrt{\frac{2 G M^{\star}}{x} \cos ^{2} \phi \sin \phi} .
$$

The line-of-sight component of the acceleration is $d v_{z} / d t=\left(d v_{z} / d x\right)(d x / d t)$ or

$$
a_{z}=\frac{v_{z}^{2}}{2 x} \tan \phi
$$

Since the accelerations of the high-velocity features are less than $0.2 \mathrm{~km} \mathrm{~s}^{-1} \mathrm{yr}^{-1}$, $\phi<7^{\circ}$. The required central mass in terms of the Keplerian mass (compare eqs. 5 and 3$)$ is

$$
M^{\star}=\frac{M}{2 \cos ^{2} \phi \sin \phi} .
$$

For $\phi=7^{\circ}, M^{\star}=1.5 \times 10^{8} M_{\odot}$. This changes the value of $R$ for the systemic features by a factor of $\left(\cos ^{2} \phi \sin \phi\right)^{1 / 3}=1.6$ to $0.21 \mathrm{pc}$, but not the estimate of the enclosed central mass density (see eq. 2 ). The relative transverse velocity of the high-velocity features is $v_{x}=v_{z} \tan \phi$ or about $200 \mathrm{~km} \mathrm{~s}^{-1}$, which corresponds to about $7 \mu \operatorname{arcsec} \mathrm{yr}^{-1}$, which is below the $15 \mu \operatorname{arcsec}$ noise threshold (set by the weak blueshifted features).

The small value of $\phi$, the angle to the line of sight, allows for a rapid change in velocity with respect to transverse distance, while keeping the lineof-sight accelerations and proper motions small. This model cannot be ruled out on the basis of measurements available now. The main argument against this model is that the material would have to be ejected at exactly the escape velocity, which seems highly unlikely. The required geometry seems implausible since the outflow would have to be nearly along the line of sight and in the plane of the disk defined by the systemic features.

\section{The High-Velocity Features as Free-Fall Motion}

The constraints are exactly the same for the infall case as for the outflow case except for a sign change. The geometry is depicted in Fig. 1 (center). The mass accretion rate is $\rho v \Omega R^{2}$, where $\rho$ is the density and $\Omega$ is the solid angle of the infalling streamer. The accuracy of the apparent Keplerian dependence (eq. 5) means that the angular size of the streamer, $\Delta \phi$, is less than $1^{\circ}$. Even with $\Delta \phi$ $=1^{\circ}$, and a standard maser density of $10^{10}$ molecules $\mathrm{cm}^{-3}$ or $3 \times 10^{-14} \mathrm{~g} \mathrm{~cm}^{-3}$, the accretion rate would be $10^{2} M_{\odot} \mathrm{yr}^{-1}$, which is unreasonably high. 


\section{Conclusion}

Since the Keplerian disk model fits the available data so beautifully, I hesitated to bring up alternate possibilities. However, it is interesting to note that the infall/outflow model can be ruled out on plausibility arguments, but not, strictly speaking, on the basis of the available measurements. Note that for the infall and outflow models, there is only a limit on the angle $\phi$ (i.e., $\phi<7^{\circ}$ ), which may grow more stringent as limits on the proper motions and accelerations of the high-velocity features are more tightly constrained.

I thank Lincoln Greenhill for help in preparing the figure.

\section{References}

Burbidge, E.M. 1996, in The Galactic Center, Proc. of the 4th ESO/CTIO Conference, ed. R. Gredel, (ASP Conference Series 102, San Francisco), p. 471 .

Greenhill, L. J., Henkel, C., Becker, R., Wilson, T.L., \& Wouterloot, J. G.A. 1995, A\&A, 304, 21.

Herrnstein, J. R. 1997, Ph.D. thesis, Harvard University.

Herrnstein, J. R., Greenhill, L. J., \& Moran, J. M. 1996, Ap.J.(Lett.), 468, L17.

Herrnstein, J.R., Moran, J.M., Greenhill, L. J., Diamond, P.J., Miyoshi, M., Nakai, N., \& Inoue, M. 1996, in The Physics of Liners in View of Recent Observations, eds. M. Eracleous, A. Koratkar, C. Leitherer, \& L. Ho, (ASP Conference Series 103, San Francisco), p. 193.

Miyoshi, M., Moran, J.M., Herrnstein, J. R., Greenhill, L. J., Nakai, N., Diamond, P. J., \& Inoue, M. 1995, Nature, 373, 127.

Moran, J. M., Greenhill, L. J., Herrnstein, J.R., Diamond, P. J., Nakai, N., Inoue, M.,\& Miyoshi, M. 1995, Proc. Nat. Acad. Sci. USA, 92, 11427.

Ozernoy, L. M. 1996, Bull. AAS, 28, 846.

Pietsch, W., Vogler, A., Kahabka, P., Jain, A., \& Klein, V. 1994, A\&A, 284, 386. 